existiert. Die Vorschrift des $\S 113$ I 2, 3 VwGO selbst ist hingegen rein prozessrechtlicher Natur und stellt keine materiell-rechtliche Grundlage für eine Rückgängigmachung der Vollzugsfolgen eines Verwaltungsakts dar. ${ }^{2406}$

Die Prüfung der Begründetheit der Anfechtungsklage kann somit nach folgendem Schema erfolgen:

I. Begründetheit des Aufhebungsantrags (§ 113 I 1 VwGO)

II. Begründetheit des Annexantrags auf Vollzugsfolgenbeseitigung (§ 113 I 2, 3 VwGO):

$\rightarrow$ Prüfung des allgemeinen Folgenbeseitigungsanspruchs nach dem unter

$\S 5$ Rn. 187 dargestellten Schema, sofern keine vorrangige, spezialgesetzliche Anspruchsgrundlage besteht.

\title{
VI. Die Begründetheitsprüfung bei der Anfechtung von Nebenbestimmungen (Christian Kaerkes)
}

Nach der herrschenden Meinung (s. ausführlich zur statthaften Klageart gegen $\mathbf{1 3 8 8}$ Nebenbestimmungen die Rn. 249ff.) ist gegen belastende Nebenbestimmungen die Anfechtungsklage statthaft. Die Klage ist dann begründet, wenn die Nebenbestimmung rechtswidrig ist und den Kläger in seinen Rechten verletzt, vgl. § 113 I 1 VwGO. Der Bürger möchte aber nur die Nebenbestimmung beseitigen, während der begünstigende Hauptverwaltungsakt (meist eine Genehmigung) bestehen bleiben soll. Dieses Ziel kann nur erreicht werden, wenn es denn möglich ist, die Nebenbestimmung von dem Hauptverwaltungsakt zu trennen („Teilbarkeit“).

Nach der Ansicht der Rechtsprechung ist für die Begründetheit der Anfechtungsklage gegen eine Nebenbestimmung deshalb zusätzlich erforderlich, dass der Hauptverwaltungsakt „sinnvoller- und rechtmäßiger Weise bestehen bleiben kann.“2407 Diese ungeschriebenen Voraussetzungen können auf die Bindung des Gerichtes an das Gesetz zurückgeführt werden, vgl. Art. 20 III GG.

Nach der materiellen Rechtmäßigkeit der Nebenbestimmung ist daher zusätzlich zu prüfen, ob der Hauptverwaltungsakt ohne die Nebenbestimmung „sinnvoll und rechtmäßig“ ist. Die Begründetheit der Anfechtungsklage setzt demnach voraus:

2406 Ahrens, Staatshaftungsrecht, 3. Aufl. 2018, Rn. 302; Detterbeck, NVwZ 2019, 97 (98).

2407 BVerwG, Urt. v. 22.11.2000, Az.: 11 C 2.00 = BVerwGE 112, 221 (224). 
- Rechtswidrigkeit der Nebenbestimmung,

- Verletzung des Klägers in dessen Rechten und

- Sinnvoller und rechtmäßiger Rest-Verwaltungsakt

1390 Für die Klausur bietet sich deshalb etwa der folgende Obersatz an: „Die Klage ist begründet, soweit die Nebenbestimmung rechtswidrig ist, der Betroffene dadurch in seinen Rechten verletzt wird und der Hauptverwaltungsakt noch rechtmäßig und sinnvollerweise bestehen bleiben kann, vgl. § 113 I 1 VwGO.“

\section{Rechtswidrigkeit der Nebenbestimmung}

1391 Die Prüfung der Rechtswidrigkeit einer Nebenbestimmung orientiert sich an dem allgemeinen Schema, das schon von der Prüfung eines normalen Verwaltungsakts bekannt ist (s. Rn. 507 ff.; 553 ff.):

- Ermächtigungsgrundlage,

- Formelle Rechtmäßigkeit und

- Materielle Rechtmäßigkeit.

Einige wichtige Besonderheiten sind aber bei den einzelnen Prüfungspunkten zu beachten: ${ }^{2408}$

\section{a) Ermächtigungsgrundlage}

1392 Nebenbestimmungen können die Freiheit des Bürgers beschränken und bedürfen daher einer gesetzlichen Grundlage (Vorbehalt des Gesetzes - s. Rn. 555 ff.). Ermächtigungsgrundlage für den Erlass einer Nebenbestimmung wird regelmäßig § 36 I VwVfG oder § 36 II VwVfG sein. Seltener kann auch eine spezialgesetzliche Regelung in Betracht kommen.

\section{b) Formelle Rechtmäßigkeit}

1393 Im Rahmen der formellen Rechtmäßigkeit ist zu prüfen, ob die Anhörung (s. Rn. 631 ff.) gem. § 28 I VwVfG ordnungsgemäß erfolgt ist. Weil Nebenbestimmungen den Bürger belasten, muss der Bürger richtigerweise auch die Möglichkeit haben, sich rechtzeitig gegenüber der Behörde zu äußern.

2408 Hufen/Bickenbach, JuS 2004, 966 ff. 
Examenswissen: Diese Ansicht ist nicht unumstritten. ${ }^{2409}$ Dahinter steht meist 1394 die Überlegung, dass die Anhörung nur für belastende Hauptverwaltungsakte notwendig sei, Nebenbestimmungen aber öfters zu begünstigenden Verwaltungsakten hinzugefügt werden.

Fehlt es an der erforderlichen Anhörung, ist die Nebenbestimmung formell $\mathbf{1 3 9 5}$ rechtswidrig. Allerdings kann der Fehler nach allgemeinen Regeln (s. Rn. 693 ff.) geheilt werden (§ $45 \mathrm{VwVfG}$ ) oder unbeachtlich sein (§ $46 \mathrm{VwVfG})$.

Im Übrigen richtet sich die formelle Rechtmäßigkeit nach dem bekannten 1396 Schema (s. Rn. 579ff.):

- Zuständigkeit,

- Verfahren und

- Form.

\section{c) Materielle Rechtmäßigkeit}

Die materielle Rechtmäßigkeit erfordert, dass die Anforderungen der Ermäch- 1397 tigungsgrundlage eingehalten werden.

\section{aa) Spezialgesetzliche Ermächtigung}

Kommt ausnahmsweise nicht $§ 36$ VwVfG, sondern eine andere Rechtsgrundlage 1398 in Betracht, ist diese als spezialgesetzliche Regelung vorrangig zu prüfen. Es müssen dann nur die Voraussetzungen dieser Rechtsgrundlage gegeben sein. Hingegen kommt es auf $\S 36$ VwVfG und dessen Voraussetzungen nicht mehr an.

\section{bb) Regelfall des $§ 36$ VwVfG}

In der überwiegenden Anzahl der Fälle wird es allerdings auf § 36 VwVfG an- 1399 kommen. Dabei ist danach zu unterscheiden, ob die Behörde beim Erlass des Hauptverwaltungsaktes über Ermessen verfügt oder nicht (s. Rn. 729ff.).

\section{(1) Gebundene Entscheidung}

Im Anwendungsbereich des § 36 I VwVfG - für einen gebundenen Hauptver- 1400 waltungsakt - ist zunächst zu prüfen, ob die Nebenbestimmung „durch Rechts-

2409 Vgl. näher Stelkens, in: Stelkens/Bonk/Sachs, VwVfG, 9. Aufl. 2018, § 36 Rn. 25 und § 28 Rn. $31 \mathrm{ff}$. 
vorschrift zugelassen ist oder [...] sie sicherstellen soll, dass die gesetzlichen Voraussetzungen des Verwaltungsakts erfüllt werden.“

In der Klausur ist insbesondere die Sicherstellung der gesetzlichen Voraussetzungen bedeutsam. Hier ist zu begutachten, ob erstens der Bürger derzeit keinen Anspruch auf den Hauptverwaltungsakt hat, weil nicht alle gesetzlichen Voraussetzungen erfüllt sind, und zweitens, ob die Nebenbestimmung die fehlende(n) Voraussetzung(en) „ersetzt“ (d.h. deren Erfüllung sicherstellt). Sind hingegen bereits alle gesetzlichen Voraussetzungen erfüllt, muss der Bürger den begehrten Hauptverwaltungsakt auch ohne Nebenbestimmungen erhalten.

\section{(2) Ermessensentscheidung}

1402 Liegt der Hauptverwaltungsakt im Ermessen der Behörde, dann sind auch Nebenbestimmungen ohne weitere Voraussetzungen grundsätzlich zulässig, vgl. § 36 II VwVfG. Es kommt insbesondere nicht darauf an, dass die gesetzlichen Voraussetzungen des Verwaltungsakts sichergestellt werden. Daher muss sehr deutlich zwischen den beiden Absätzen des §36 VwVfG unterschieden werden: - Falls ein Anspruch auf den Hauptverwaltungsakt besteht, dann I.

- Falls der Hauptverwaltungsakt im Ermessen steht, dann II.

\section{(3) Weitere Voraussetzungen}

1403 Der Erlass von Nebenbestimmungen ist eine Ermessensentscheidung der Behörde (,darf“, „nach pflichtgemäßem Ermessen“, vgl. § 36 I, II VwVfG). Die Behörde kann zwar Nebenbestimmungen erlassen, muss es aber nicht. Diese Ausübung des Ermessens darf nicht mit dem Ermessen zum Erlass des Hauptverwaltungsaktes verwechselt werden. In der Klausur ist die Entscheidung der Behörde auf Ermessensfehler zu überprüfen (s. zur Ermessensfehlerlehre Rn. 739 ff.). Dabei sind auch die Grundrechte und der Grundsatz der Verhältnismäßigkeit zu berücksichtigen.

1404 Soweit entsprechende Anhaltspunkte bestehen, ist auch auf § 36 III VwVfG einzugehen. Das Kopplungsverbot (s. Rn. $243 \mathrm{ff}$.) ist dann nicht verletzt, wenn ein sachlicher Zusammenhang zwischen der Nebenbestimmung und dem Hauptverwaltungsakt besteht.

1405 Im Ergebnis setzt sich also die Prüfung der materiellen Rechtmäßigkeit zusammen aus:

- Einhaltung der gesetzlichen Voraussetzungen des §36 VwVfG,

- Ordnungsgemäße Ausübung des Ermessens und

- Ggf. Beachtung des Kopplungsverbotes. 


\section{Verletzung subjektiver Rechte}

Voraussetzung für den Erfolg der Anfechtungsklage ist weiterhin, dass der Kläger 1406 in seinen Rechten verletzt wird, vgl. § 113 I 1 VwGO (s. ausführlich § 2 Rn. 828ff.). Für Nebenbestimmungen gilt, dass für rechtswidrige, belastende Nebenbestimmungen eine solche Rechtsverletzung anzunehmen ist. Der Kläger hat nämlich entweder einen Anspruch auf einen nebenbestimmungsfreien Verwaltungsakt oder einen Anspruch auf ermessensfehlerfreie Entscheidung über den Erlass der Nebenbestimmungen. ${ }^{2410}$

Im Gutachten reicht deshalb regelmäßig die Feststellung aus, dass die rechtswidrigen Nebenbestimmungen den Kläger auch in dessen Rechten verletzen, vgl. § 113 I 1 VwGO.

\section{Sinnvoller und rechtmäßiger Rest-Verwaltungsakt}

Der verbleibende Verwaltungsakt wird regelmäßig sinnvoll sein. Der Rest-Ver- 1407 waltungsakt wäre dann sinnlos, wenn entweder „der verbleibende Teil keine selbstständige Bedeutung hat oder der Verwaltungsakt bei Wegfall der Nebenbestimmung einen anderen Sinn erhalten und dadurch seinen Zweck verfehlen würde.“2411 Da Verwaltungsakte ohne Nebenbestimmungen erlassen werden können, ist aber kaum einzusehen, wann die Aufhebung einer Nebenbestimmung den Verwaltungsakt einmal sinnlos werden lassen sollte. ${ }^{2412}$

Wichtiger ist das Kriterium der Rechtmäßigkeit des Rest-Verwaltungsakts. 1408 Dieser wird insbesondere rechtswidrig sein, wenn die Nebenbestimmung erst die Rechtmäßigkeit des Hauptverwaltungsaktes herbeiführen sollte, vgl. §36 I VwVfG. ${ }^{2413}$ Ohne die Nebenbestimmung wäre die Erfüllung der gesetzlichen Voraussetzungen nicht mehr sichergestellt.

Beispiel: Baugenehmigung mit der Auflage, den Brandschutz zu gewährleisten

Eine Baugenehmigung darf regelmäßig nur dann erteilt werden, wenn das Vorhaben mit allen öffentlich-rechtlichen Vorschriften im Einklang steht. Zu diesen Vorschriften gehört auch der Brandschutz, sodass das Vorhaben eigentlich nicht genehmigungsfähig wäre. Die Rechtmäßigkeit der Baugenehmigung kann nur dadurch herbeigeführt werden, dass eine Nebenbestimmung erlassen wird.

2410 Hufen/Bickenbach, JuS 2004, 966 (968).

2411 Sproll, NJW 2002, 3221 (3222).

2412 Vgl. Bumke, in: Festschrift für Battis, 2014, 177 (194).

2413 Vgl. Bumke, in: Festschrift für Battis, 2014, 177 (187). 
Entfällt die Nebenbestimmung, dann kann die Baugenehmigung nicht mehr rechtmäßiger Weise bestehen bleiben. Es stünden dem Vorhaben wieder öffentlich-rechtliche Vorschriften entgegen. In einem solchen Fall kann die Nebenbestimmung nicht aufgehoben werden. Die Anfechtungsklage wäre zwar zulässig, aber unbegründet.

1409 Der Rest-Verwaltungsakt kann schließlich deshalb rechtswidrig sein, weil die Behörde ihr Ermessen einheitlich ausgeübt hat (Hauptverwaltungsakt und Nebenbestimmung). Wird die Nebenbestimmung aufgehoben, konnte die Behörde zuvor noch keine selbstständige Ermessensentscheidung über den verbleibenden Rest-Verwaltungsakt treffen. ${ }^{2414}$

Beispiele: Entscheidung über eine Subvention mit einer (belastenden) Auflage; Befristete Sondernutzungserlaubnis für eine künstlerische Darbietung

Die Behörde hat über Hauptverwaltungsakt und Nebenbestimmung bisher ausschließlich gemeinsam „nachgedacht“. Das ihr zustehende Ermessen hat die Behörde nicht für den Fall einer Subvention ohne Auflage (oder Sondernutzungserlaubnis ohne Befristung) ausgeübt. Deshalb könnte man den Rest-Verwaltungsakt für rechtswidrig und die Anfechtungsklage für unbegründet erachten.

Zusammengefasst existieren also verschiedene Fallgruppen, wann ein Hauptverwaltungsakt nicht „rechtmäßiger Weise bestehen bleiben“ kann:

- Herbeiführung der Rechtmäßigkeit gerade durch die Nebenbestimmung,

- Einheitliche Ermessensausübung der Behörde und

- Sonstige Fälle der Rechtswidrigkeit.

Erweist sich der verbleibende Verwaltungsakt nicht als „rechtmäßig und sinnvoll“, dann ist die Anfechtungsklage zulässig, aber unbegründet.

Dem Bürger steht allerdings immer noch die Verpflichtungsklage zur Verfügung. Wenn der begehrte Verwaltungsakt rechtswidrig ist, weil die gesetzlichen Voraussetzungen ohne die Nebenbestimmung nicht sichergestellt sind, kann auch die Verpflichtungsklage keinen Erfolg haben. Der Bürger hat keinen Anspruch auf den Erlass eines rechtswidrigen Verwaltungsakts. Denkbar ist aber ein Anspruch auf den Verwaltungsakt mit einer rechtmäßigen Nebenbestimmung. 1412 Der Rest-Verwaltungsakt kann außerdem deshalb rechtswidrig sein, weil die Ermessensausübung nur einheitlich erfolgt ist. Da der Verwaltungsakt in diesem Fall im Ermessen der Behörde liegt, steht dem Bürger nach allgemeinen Regeln

2414 Schenke, in: Festschrift für Roellecke, 1997, 281 (295). 
nur ein Anspruch auf eine ermessensfehlerfreie Entscheidung zu. Im Ergebnis erreicht der Bürger regelmäßig ein „Bescheidungsurteil“, vgl. § 113 V 2 VwGO.

In der Klausur darf daher die Prüfung der Verpflichtungsklage nicht vergessen werden, wenn die Anfechtungsklage daran scheitert, dass der Rest-Verwaltungsakt nicht „rechtmäßig und sinnvoll“ wäre (zur Prüfung der Verpflichtungsklage ausführlich $\S 3$ ).

Examenswissen: Die zusätzliche Voraussetzung, dass der Verwaltungsakt rechtmäßig bestehen bleiben können muss, ist in der Literatur nicht unumstritten. Die Bindung an Gesetz und Recht (Art. 20 III GG) hilft in diesem Fall nicht weiter („Rechtsstaatsklemme“ $)^{2415}$, weil entweder die rechtswidrige Nebenbestimmung hingenommen werden muss oder ein rechtswidriger Verwaltungsakt geschaffen wird.

Letztendlich geht es um die Frage, ob die Nachteile durch den Bürger oder die Verwaltung getragen werden sollten. Hierauf sind zwei Antworten möglich:

Widersprüchliches Verhalten des Bürgers oder Verantwortung der Behörde: Der Bürger muss sich vorhalten lassen, dass er die Aufhebung des rechtswidrigen Zustandes nur insoweit begehrt, als er belastet ist. Die rechtswidrige Begünstigung möchte er weiterhin in Anspruch nehmen können. Darin kann ein widersprüchliches Verhalten erblickt werden. ${ }^{2416}$ Der Bürger verlangt nicht, den rechtswidrigen Zustand zu beenden, sondern nur, ihn zu seinen Gunsten zu verändern und zu perpetuieren.

Der Behörde ist hingegen der Vorwurf zu machen, dass sie für die anfängliche Rechtswidrigkeit verantwortlich ist. Immerhin hat die Behörde den Verwaltungsakt mit der rechtswidrigen Nebenbestimmung überhaupt erlassen. Weil rechtswidrige Verwaltungsakte aufgehoben werden können (§§ 48, 49 VwVfG), könnte man es der Behörde auch anlasten, wenn sie von dieser Möglichkeit keinen Gebrauch macht.

Wer diese Verantwortung der Behörde betont, kann die in der Rechtsprechung aufgestellte Voraussetzung (,Rechtmäßigkeit des Rest-Verwaltungsakts“) ablehnen. Zu prüfen sind dann „nur“ die Voraussetzungen des § 113 I 1 VwGO. Die Anfechtungsklage ist begründet, soweit die Nebenbestimmung rechtswidrig ist und den Kläger in seinen Rechten verletzt.

\section{Literaturhinweise}

Hufen/Bickenbach, JuS 2004, 966: „Der Rechtsschutz gegen Nebenbestimmungen 1415 zum Verwaltungsakt“; Im Übrigen s. bereits Rn. 279

2415 Hufen/Bickenbach, JuS 2004, 966 (967).

2416 Schenke, JuS 1983, 182 (185). 